\title{
Prevalência e fatores associados à polifarmácia excessiva em pessoas idosas institucionalizadas do Sul do Brasil
}

\author{
Prevalence and factors associated with excessive polypharmacy in institutionalized older people in \\ southern Brazil
}

\author{
Andréia Mascarelo' ${ }^{1}$ \\ Emanuelly Casal Bortoluzzil $\mathbb{D}$ \\ Siomara Regina Hahn² $\mathbb{D}$ \\ Ana Luisa Sant'Anna Alves' $\mathbb{D}$ \\ Marlene Doring' (ID \\ Marilene Rodrigues Portella' $\mathbb{D}$
}

\section{Resumo}

Objetivo: Verificar a prevalência e os fatores associados à polifarmácia excessiva em pessoas idosas institucionalizadas. Método: Estudo transversal com 478 pessoas idosas residentes em instituições de longa permanência para idosos. A variável dependente foi polifarmácia excessiva, definida como o uso concomitante de dez ou mais medicamentos. As variáveis independentes incluíram informações sociodemográficas e de saúde. Utilizou-se a regressão de Poisson com variância robusta para analisar o efeito das variáveis independentes em relação ao desfecho. Resultados: A prevalência de polifarmácia excessiva foi de 29,3\%, associada à cardiopatia ( $\mathrm{RP}=1,40 ; \mathrm{IC} 95 \% 1,03-1,91)$, diabetes mellitus ( $\mathrm{RP}=1,52 ; \mathrm{IC} 95 \%$ 1,15-2,01), depressão ( $\mathrm{RP}=1,42$; IC95\% 1,08-1,87), internação hospitalar no último ano $(\mathrm{RP}=1,36$; IC95\% 1,02-1,80) e ao uso de medicamento potencialmente inapropriado para idosos (RP=2,13; IC95\% 1,60-2,83). Conclusão: A polifarmácia excessiva foi frequente entre pessoas idosas institucionalizadas. Os resultados sugerem que as doenças prevalentes entre pessoas idosas, a hospitalização e o uso de medicamentos potencialmente inapropriados são fatores para o uso de polifarmácia excessiva nessa população. Esses achados podem instruir ações com vistas à otimização da farmacoterapia prescrita às pessoas idosas.

\section{Abstract}

Objective: To verify the prevalence and factors associated with excessive polypharmacy in institutionalized older people. Method: Cross-sectional study with 478 older people living in long-term care facilities. The dependent variable was excessive polypharmacy

\footnotetext{
Universidade de Passo Fundo (UPF), Programa de Pós-Graduação em Envelhecimento Humano. Passo Fundo, RS, Brasil

2 Universidade de Passo Fundo (UPF), Instituto de Ciências Biológicas, Curso de Farmácia. Passo Fundo, RS, Brasil.
}

Financiamento da pesquisa: Programa Nacional de Cooperação Acadêmica (PROCAD). $\mathrm{N}^{\circ}$ do processo: 88881.068447/2014-01, convênio PROCAD/CAPES 2972/14. O presente trabalho foi realizado com apoio da Coordenação de Aperfeiçoamento de Pessoal de Nível Superior - Brasil (CAPES) - Código de Financiamento 001

Os autores declaram não haver conflito na concepção deste trabalho.

\section{Palavras-chave:}

Polimedicação. Idoso. Instituição de Longa Permanência para Idosos. Saúde do Idoso. Estudos Transversais. 
which is defined as the concomitant use of ten or more medications. The independent variables included sociodemographic and health information. The Poisson regression with robust variance was used to analyze the effect of the independent variables compared to the outcome. Results: The prevalence of excessive polypharmacy was $29.3 \%$ associated with heart disease ( $\mathrm{PR}=1.40$; 95\%CI 1.03-1.91), diabetes mellitus ( $\mathrm{PR}=1.52$; 95\%CI 1.15$2.01)$, depression ( $\mathrm{PR}=1.42$; 95\% CI 1.08-1.87), hospitalization in the last year ( $\mathrm{PR}=1.36$; $95 \%$ CI 1.02-1.80), and the use of potentially inappropriate medication for older people $(\mathrm{PR}=2.13$; 95\%CI 1.60-2.83). Conclusion: Excessive polypharmacy was frequent among institutionalized older people. The results suggest that prevalent diseases among older people, hospitalization, and the use of potentially inappropriate medications are reasons for the use of excessive polypharmacy by this population. Said findings can guide actions aimed at optimizing the pharmacotherapy prescribed to older people.
Keywords: Polypharmacy. Elderly. Homes for the Aged. Health of the Elderly. CrossSectional Studies.

\section{INTRODUÇÃO}

As doenças crônicas e múltiplas tendem a se manifestar com frequência no grupo etário idoso, contribuindo para a utilização de polifarmácia nessa população ${ }^{1,2}$. Um ou mais fármacos podem ser utilizados para tratar cada doença ou sintoma ${ }^{2,3}$ levando a esquemas terapêuticos complexos ${ }^{3,4}$.

A polifarmácia é um problema de saúde pública global ${ }^{5}$ e um dos maiores desafios relacionados à população que envelhece, com ônus para pessoas idosas, famílias e sistemas de saúde ${ }^{6}$. Em todo o mundo, as taxas de prescrição de medicamentos apresentam tendência crescente devido ao aumento da população idosa e à disponibilidade de fármacos ${ }^{2,6}$. A prevalência de polifarmácia relatada na literatura varia de $10 \%$ até cerca de $90 \%$, em estudos que consideram diferentes faixas etárias, definições de polifarmácia e localizações geográficas ${ }^{6}$.

Não há consenso na literatura acerca da definição de polifarmácia e polifarmácia excessiva. Contudo, a maioria dos estudos utiliza o critério numérico, ${ }^{2,6}$, considerando polifarmácia o uso simultâneo de cinco ou mais medicamentos ${ }^{7,8} \mathrm{e}$ polifarmácia excessiva $\mathrm{O}$ uso concomitante de 10 ou mais medicamentos ${ }^{4,9}$.

A polifarmácia excessiva não denota, por si só, polifarmácia inadequada ${ }^{2,3}$. O uso de vários medicamentos não é necessariamente imprudente $\mathrm{e}$, em alguns casos, pode ser necessário e benéfico ${ }^{2,5}$. No entanto, embora uma combinação adequada de fármacos em pacientes com problemas de saúde complexos possa melhorar sua condição clínica ${ }^{2}$, quanto maior o número de medicamentos em uso, maiores são os riscos para o idoso ${ }^{1}$, pois se elevam as chances de efeitos adversos e interações medicamentosas ${ }^{2}$.

As doenças crônicas, o estado funcional e as alterações fisiológicas próprias do envelhecimento causam mudanças, tanto na ação do organismo sobre os fármacos como dos fármacos sobre o organismo. A redução da água corporal, da albumina sérica e do fluxo sanguíneo hepático e renal, assim como, o aumento da gordura corporal são exemplos de transformações fisiológicas que levam à alteração do volume, concentração e distribuição dos medicamentos ${ }^{10}$.

Nenhum fármaco é completamente seguro para uso em idosos, visto que as propriedades farmacocinéticas e farmacodinâmicas de muitos deles não são completamente conhecidas nesse grupo, pois pessoas idosas, frágeis e com multimorbidade são comumente excluídas de pesquisas necessárias à aprovação de novos medicamentos. Contudo, esse é um grupo visto com frequência na prática clínica e mais sujeito à polifarmácia ${ }^{2}$.

Nesse sentido, a população idosa institucionalizada merece maior atenção, pois geralmente são mais frágeis, possuem maior número de doenças crônicas e usam mais medicamentos em comparação com pessoas idosas da comunidade ${ }^{1-4}$ estando assim mais susceptíveis aos riscos de complicações associadas à polifarmácia ${ }^{2}$. Estudos apontam que o número médio de medicamentos utilizados, assim como a prevalência de polifarmácia excessiva é maior entre pessoas idosas institucionalizadas. Investigações anteriores reportaram prevalências de polifarmácia 
excessiva entre pessoas idosas que residem em instituições, de $16,8 \%{ }^{3}$ até $44,1 \%{ }^{11}$, o que demanda especial atenção a esse segmento ${ }^{3,11}$. Fatores como usar 10 ou mais medicamentos e residir em um lar de idosos podem aumentar a probabilidade de um idoso sofrer danos relacionados à medicação ${ }^{2}$.

Em alguns países, sobretudo nos desenvolvidos, o estudo dessa temática está bem estabelecido ${ }^{3,4}$. No entanto, no Brasil, não foi encontrada publicação acerca da prevalência e dos fatores associados à polifarmácia excessiva entre pessoas idosas que residem em instituições de longa permanência para idosos (ILPI), após extensa busca na base de dados MEDLINE/Pubmed, utilizando os descritores "Polypharmacy" and "Aged" and "Homes for the Aged", nos últimos 10 anos.

Diante disso, o presente estudo teve por objetivo verificar a prevalência e os fatores associados à polifarmácia excessiva entre pessoas idosas institucionalizadas.

\section{MÉTODO}

Trata-se de um estudo de corte transversal, com pessoas de 60 anos de idade ou mais residentes em Instituições de Longa Permanência para Idosos (ILPI). Este estudo é um recorte da pesquisa Padrões de envelhecimento e longevidade: aspectos biológicos, educacionais e psicossociais, desenvolvida sob coordenação da Universidade Estadual de Campinas (UNICAMP), São Paulo (SP) e participação da Universidade Católica de Brasília (UCB), Distrito Federal (DF) e Universidade de Passo Fundo (UPF), Rio Grande do Sul (RS). Os dados foram coletados no ano de 2017 em 19 ILPI localizadas nos municípios de Passo Fundo (RS), Carazinho (RS) e Bento Gonçalves (RS), Brasil.

Foram incluídas no estudo todas as ILPI dos municípios de Passo Fundo (RS), Carazinho (RS) e Bento Gonçalves (RS), localizados nas regiões Norte e Serra do estado do RS, pela necessidade de agregar uma amostra maior de pessoas idosas institucionalizadas. A escolha dos municípios obedeceu a critérios como proximidade geográfica e características semelhantes em relação à proporção de pessoas idosas na população e distribuição por sexo e faixa etária. A população estimada para o ano de 2015, no município de Passo Fundo (RS), era de 196.741 habitantes, em Carazinho (RS) de 62.037 habitantes e em Bento Gonçalves (RS) de 113.287 habitantes e a respectiva proporção de pessoas idosas na população era de 13,58\%, 16,13\% e 14,11\%. Entre as pessoas idosas, em cada localidade, a maioria era de mulheres $(58,2 \%, 58,1 \%$ e $56,1 \%)$ e a proporção de pessoas com 80 anos e mais era de 13,9\%, 14,6\% e $14,1 \%$, respectivamente ${ }^{12}$.

Das 35 ILPI localizadas nos municípios selecionados, todas foram convidadas a participar da pesquisa, entretanto, 19 manifestaram seu aceite, das quais, $14(58,3 \%)$ se localizavam no município de Passo Fundo (RS), onde residiam 281 pessoas idosas, 1 (50,0\%) no município de Carazinho (RS) com 92 pessoas idosas e $4(44,4 \%)$ no município de Bento Gonçalves (RS), nas quais viviam 106 pessoas idosas. A população total residente nessas instituições era de 479 pessoas idosas. Após o aceite por parte das ILPI, as pessoas idosas e os seus responsáveis foram convidados a participar do estudo. Foram incluídos todos os indivíduos com idade igual ou superior a 60 anos e excluídos aqueles que no momento da entrevista estavam internados em hospital ou que não foram localizados após três tentativas dos entrevistadores, em dias e horários alternados.

Para o cálculo da amostra levou-se em consideração um nível de confiança de $95 \%$, poder estatístico de $80 \%$, razão de não exposto: exposto (presença de Doença Crônica Não Transmissível) de 2, prevalência do desfecho no grupo exposto de $30 \% \%^{1,4,9,11}$, totalizando 291 idosos e acrescentou-se $20 \%$ para perdas e recusas $(n=349)$. Entretanto, por tratar-se de um estudo com diferentes desfechos, optou-se por investigar todas as pessoas idosas residentes em ILPI dos municípios selecionados que preenchiam os critérios de inclusão. Constituíram a amostra do estudo 478 pessoas idosas.

Considerou-se como variável dependente a polifarmácia excessiva, definida como o uso concomitante de 10 ou mais medicamentos, prescritos nos três meses que antecederam a pesquisa. $\mathrm{O}$ uso de medicamentos foi verificado junto ao prontuário das pessoas idosas e as substâncias ativas listadas, conforme quinto nível da Classificação Anatômica Terapêutica Química (ATC) ${ }^{13}$. 
As variáveis independentes, definidas com base em investigações anteriores ${ }^{1,4,9,11,14}$, incluíram informações sociodemográficas e relativas à ILPI: tipo de ILPI (privada /filantrópica), idade (em anos), longevidade considerada idade maior ou igual a 80 anos (longevo/não longevo), sexo (masculino/ feminino), cor/raça (branco/não branco), estado civil (com companheiro/sem companheiro), tempo de internação (em meses), escolaridade (frequentou/não frequentou a escola) e visita de familiares (sim/não);

As variáveis relacionadas à saúde incluíram: cardiopatia (sim/não), hipertensão arterial sistêmica (sim/não), acidente vascular encefálico (sim/não), diabetes mellitus (sim/não), doença pulmonar (sim/ não), depressão (sim/não), demência (sim/não), dor crônica nos últimos seis meses (sim/não), multimorbidade (sim/não), uso de Medicamento Potencialmente Inapropriado para Idosos (MPI) (sim/não) e a ocorrência nos últimos 12 meses de incontinência urinária (sim/não), incontinência fecal (sim/não), insônia (sim/não), internação hospitalar (sim/não) e número de internações hospitalares (número). As variáveis relacionadas à funcionalidade foram: estado cognitivo (com declínio cognitivo/ sem declínio cognitivo) e atividades básicas da vida diária (ABVD) (independente/dependente).

A multimorbidade foi caracterizada pela presença de duas ou mais condições crônicas em um indivíduo. Como a lista de morbidades para sua operacionalização ainda não está definida para o contexto brasileiro, foram incluídas, para a construção da variável, condições que apresentam uma ou mais das seguintes características: causam alterações patológicas não reversíveis no organismo, geram incapacidade residual, são permanentes, indicam a necessidade de reabilitação ou de cuidados de longa duração ${ }^{15}$. Assim as morbidades incluídas foram a presença de cardiopatia, de hipertensão arterial sistêmica, de diabetes mellitus, de reumatismo, de doença pulmonar, de depressão, de osteoporose, de demência, de Doença de Parkinson, de sarcopenia e de fragilidade.

Para a variável uso de MPI utilizou-se os critérios de Beers $2015^{16}$. Para avalição do estado cognitivo utilizou-se o Miniexame de Estado Mental (MEEM) com pontos de corte sugeridos por Bertolucci et a ${ }^{17}: 13$ para analfabetos, 18 para baixa e média escolaridade e 26 para alta escolaridade. A partir do escore obtido, o idoso foi classificado (com declínio cognitivo/sem declínio cognitivo).

Para as ABVD a pessoa idosa foi classificada como independente ou dependente, segundo o Índice de $\mathrm{Katz}^{18}$. Aquelas que conseguiam realizar uma ou mais atividades somente com auxílio, foram classificadas como dependentes?.

As informações sociodemográficas, de saúde e uso de medicamentos foram obtidas junto ao prontuário e as relativas à funcionalidade diretamente com as pessoas idosas, em ambiente individualizado, designado pelo responsável pela ILPI.

Para minimizar inconsistências na coleta dos dados, os 4 entrevistadores, acadêmicos de enfermagem e fisioterapia, foram submetidos a um programa de treinamento e eram acompanhados por dois professores pesquisadores, responsáveis pelo estudo.

As variáveis categóricas foram apresentadas empregando-se distribuições de frequências univariadas e tabelas de contingência bi e multivariadas. As variáveis quantitativas foram descritas mediante medidas de tendência central ou posição e variabilidade e a normalidade foi verificada por meio do teste de Kolmogorov-Smirnov. Para avaliar a associação entre polifarmácia excessiva e as variáveis independentes categóricas, aplicou-se o teste qui-quadrado de Pearson. Para comparações entre os grupos das variáveis independentes quantitativas em relação à variável dependente utilizou-se o teste não paramétrico U de Mann Whitney. O nível de significância adotado foi de 5\%. Para as análises multivariadas utilizou-se a regressão de Poisson com variância robusta, estimando-se as razões de prevalência bruta e ajustada e calculando-se os respectivos intervalos de confiança de 95\%. No modelo múltiplo, foram consideradas as variáveis que tiveram um valor de $p \leq 0,20$ na análise bivariada e permaneceram no modelo aquelas $\operatorname{com} p<0,05$.

A pesquisa foi aprovada pelo Comitê de Ética em Pesquisa da Universidade de Passo Fundo (UPF), parecer número 2.097.278, em acordo com a Resolução 466/2012 do Conselho Nacional de 
Saúde. Todos os participantes do estudo assinaram o Termo de Consentimento Livre e Esclarecido.

\section{RESULTADOS}

Participaram do estudo 478 pessoas idosas com média de idade de 80,3 ( $(9,77)$ anos, com variação mínima de 60 e máxima de 109 anos. Houve predomínio de mulheres $(71,1 \%)$, de pessoas longevas $(57,0 \%)$, de escolarizados $(83,5 \%)$ e dos que residiam em ILPI filantrópicas (57,1\%), conforme Tabela 1.
Tiveram um tempo médio de permanência na ILPI de 50,53 $( \pm 73,46)$ meses e o número médio de medicamentos utilizados foi 7,40 $( \pm 3,65)$, com variação mínima de 1 e máxima de 22 medicamentos. Apenas 1,5\% não fazia uso de medicamentos.

Verificou-se elevada prevalência de declínio cognitivo $(73,2 \%)$ e dependência para ABVD $(85,0 \%)$ na população estudada. A doença crônica mais prevalente foi a hipertensão arterial sistêmica $(54,8 \%)$ e $59,6 \%$ apresentavam multimorbidade (Tabela 2).

Tabela 1. Características sociodemográficas e relativas à Instituição de Longa Permanência para Idosos, de pessoas idosas institucionalizadas (n=478). Passo Fundo, Carazinho e Bento Gonçalves, RS, Brasil, 2017.

\begin{tabular}{llc}
\hline Variável & $\mathrm{n}(\%)$ & $\mathrm{IC} 95 \%{ }^{*}$ \\
\hline Tipo de ILPI & $273(57,1)$ & $52,3-61,5$ \\
Filantrópica & $205(42,9)$ & $38,5-47,7$ \\
Privada & & \\
\hline Longevidade & $205(43,0)$ & $38,8-47,2$ \\
Não longevo & $272(57,0)$ & $52,8-61,2$ \\
Longevo & & \\
Sexo & $138(28,9)$ & $25,1-33,3$ \\
Masculino & $340(71,1)$ & $66,7-74,9$ \\
Feminino & & $84,7-90,6$ \\
Visita de familiares & $411(87,4)$ & $9,4-15,3$ \\
Sim & $59(12,6)$ & $86,7-92,0$ \\
Não & & $8,0-13,3$ \\
Cor & $424(89,5)$ & \\
Branco & $50(10,5)$ & $4,2-8,4$ \\
Não branco & & $91,6-95,8$ \\
\hline Situação conjugal & $30(6,3)$ & $80,3-87,0$ \\
Com cônjuge & $445(93,7)$ & \\
Sem cônjuge & $76(16,5)$ & \\
\hline Escolaridade & $386(83,5)$ & \\
Não frequentou a escola & & \\
Frequentou a escola & & \\
\hline
\end{tabular}

ILPI: Instituição de Longa Permanência para Idosos; *IC: Intervalo de Confiança. Os totais de frequência absoluta divergem em razão de dados faltantes. Longevidade $\mathrm{n}$ válido $=477$; Visita de familiares $\mathrm{n}$ válido $=470$; Cor $\mathrm{n}$ válido $=474$; Situação conjugal $\mathrm{n}$ válido $=475$; Escolaridade $\mathrm{n}$ válido $=462$. 
Tabela 2. Características de saúde e uso de medicamentos de pessoas idosas institucionalizadas ( $\mathrm{n}=478$ ). Passo Fundo, Carazinho e Bento Gonçalves, RS, Brasil, 2017.

\begin{tabular}{|c|c|c|}
\hline Variável & $\mathrm{n}(\%)$ & IC* $95 \%$ \\
\hline \multicolumn{3}{|c|}{ Estado cognitivo } \\
\hline Com declínio & $349(73,2)$ & $69,0-77,4$ \\
\hline Sem declínio & $128(26,8)$ & $22,6-31,0$ \\
\hline \multicolumn{3}{|l|}{ Cardiopatia } \\
\hline Sim & $84(17,8)$ & $14,4-21,4$ \\
\hline Não & $389(82,2)$ & $78,6-85,6$ \\
\hline \multicolumn{3}{|c|}{ Hipertensão arterial } \\
\hline Sim & $259(54,8)$ & $49,9-59,2$ \\
\hline Não & $214(45,2)$ & $40,8-50,1$ \\
\hline \multicolumn{3}{|c|}{ Acidente vascular encefálico } \\
\hline Sim & $103(21,6)$ & $18,1-26,1$ \\
\hline Não & $373(78,4)$ & $73,9-81,9$ \\
\hline \multicolumn{3}{|l|}{ Diabetes mellitus } \\
\hline $\operatorname{Sim}$ & $98(20,6)$ & $17,2-24,4$ \\
\hline Não & $378(79,4)$ & $75,6-82,8$ \\
\hline \multicolumn{3}{|l|}{ Depressão } \\
\hline Sim & $176(37,4)$ & $32,9-42,5$ \\
\hline Não & $295(62,6)$ & $57,5-67,1$ \\
\hline \multicolumn{3}{|l|}{ Demência } \\
\hline Sim & $236(49,8)$ & $44,5-54,2$ \\
\hline Não & $238(50,2)$ & $45,8-55,5$ \\
\hline \multicolumn{3}{|c|}{ Incontinência urinária } \\
\hline Sim & $309(65,2)$ & $61,0-69,6$ \\
\hline Não & $165(34,8)$ & $30,4-39,0$ \\
\hline \multicolumn{3}{|c|}{ Internação hospitalar } \\
\hline Sim & $148(31,5)$ & $27,0-36,4$ \\
\hline Não & $322(68,5)$ & $63,6-73,0$ \\
\hline \multicolumn{3}{|l|}{ Dor crônica } \\
\hline Sim & $167(36,6)$ & $31,4-41,5$ \\
\hline Não & $289(63,4)$ & $58,5-68,6$ \\
\hline \multicolumn{3}{|l|}{ Insônia } \\
\hline Sim & $156(33,3)$ & $29,1-37,8$ \\
\hline Não & $312(66,7)$ & $62,2-70,9$ \\
\hline \multicolumn{3}{|c|}{ Medicamento Potencialmente Inapropriado } \\
\hline Sim & $158(35,3)$ & $31,1-39,4$ \\
\hline Não & $289(64,7)$ & $60,6-68,9$ \\
\hline \multicolumn{3}{|c|}{ Atividades básicas da vida diária } \\
\hline Independente & $69(15,0)$ & $12,1-18,4$ \\
\hline Dependente & $392(85,0)$ & $81,6-87,9$ \\
\hline \multicolumn{3}{|c|}{ Polifarmácia excessiva } \\
\hline Sim & $140(29,3)$ & $25,3-33,7$ \\
\hline Não & $338(70,7)$ & $66,3-74,7$ \\
\hline \multicolumn{3}{|l|}{ Multimorbidade } \\
\hline $\operatorname{Sim}$ & $283(59,6)$ & $55,4-64,2$ \\
\hline Não & $192(40,4)$ & $35,8-44,6$ \\
\hline
\end{tabular}

MEEM: Miniexame de Estado Mental; ${ }^{*}$ IC: Intervalo de Confiança. Os totais de frequência absoluta divergem em razão de dados faltantes. Estado cognitivo n válido $=477$; Cardiopatia n válido $=473$; Hipertensão arterial $n$ válido $=473$; Acidente vascular encefálico $n$ válido $=476$; Diabetes mellitus $\mathrm{n}$ válido $=476$; Depressão $\mathrm{n}$ válido $=471$; Demência $\mathrm{n}$ válido $=474 ;$ Incontinência urinária $\mathrm{n}$ válido $=474 ;$ Internação hospitalar $\mathrm{n}$ válido $=470$; Dor crônica $n$ válido $=456$; Insônia $n$ válido $=468 ;$ MPI $n$ válido $=447$; Atividades básicas da vida diária $n$ válido $=461 ;$ Multimorbidade $n$ válido $=475$. 
Polifarmácia excessiva foi observada em 29,3\% das pessoas idosas. Verificou-se maior prevalência de polifarmácia excessiva em residentes de ILPI privadas $(33,7 \%)$, no sexo feminino $(32,6 \%)$, entre os que faziam uso de MPI $(45,6 \%)$, que possuíam diagnóstico de cardiopatia $(44,0 \%)$, de diabetes mellitus (41,8\%), de doença pulmonar $(40,0 \%)$ e de depressão (38,6\%) (Tabela 3).

Os principais grupos de medicamentos responsáveis pela polifarmácia excessiva, na população estudada, foram aqueles com ação sobre aparelho digestivo e metabolismo (95,4\%), seguidos pelos que agem sobre o sistema nervoso $(88,5 \%)$. Em cada um dos grupos, as substâncias usadas com mais frequência foram, respectivamente, o omeprazol $(53,4 \%)$ e a quetiapina $(40,5 \%)$.

Neste estudo, idade, tempo de internação na ILPI e número de internações hospitalares não apresentaram relação com polifarmácia excessiva $(p>0,05)$.

A polifarmácia excessiva apresentou associação na análise bruta com sexo $(p=0,011)$, cardiopatia ( $p=0,001)$, hipertensão arterial sistêmica $(p=0,003)$, diabetes mellitus ( $p=0,002)$, depressão $(p=0,001)$, incontinência urinária $(p=0,016)$, internação hospitalar no último ano $(p=0,008)$ e uso de MPI $(p<0,001)$ (Tabela 3).

Tabela 3. Prevalência de polifarmácia excessiva e fatores associados em pessoas idosas institucionalizadas $(\mathrm{n}=478)$. Passo Fundo, Carazinho e Bento Gonçalves, RS, Brasil, 2017.

\begin{tabular}{|c|c|c|c|c|}
\hline Variável & $\mathrm{n}(\%)$ & $p^{*}$ & RP** (IC 95\%) & $\mathrm{RP}^{* * *}(\mathrm{IC} 95 \%)$ \\
\hline Sexo & & 0,011 & & --- \\
\hline Feminino & $111(32,6)$ & & $0,69(0,47-1,00)$ & \\
\hline Masculino & $29(21,0)$ & & 1,00 & \\
\hline Tipo de ILPI & & 0,069 & & --- \\
\hline Filantrópica & $71(26,0)$ & & $1,21(0,90-1,63)$ & \\
\hline Privada & $69(33,7)$ & & 1,00 & \\
\hline Cardiopatia & & 0,001 & & \\
\hline $\operatorname{Sim}$ & $37(44,0)$ & & $1,70(1,24-2,33)$ & $1,40(1,03-1,91)$ \\
\hline Não & $101(26,0)$ & & 1,00 & 1,00 \\
\hline Hipertensão arterial & & 0,003 & & --- \\
\hline Sim & $90(34,7)$ & & $1,74(1,25-2,43)$ & \\
\hline Não & $48(22,4)$ & & 1,00 & \\
\hline Diabetes mellitus & & 0,002 & & \\
\hline Sim & $41(41,8)$ & & $1,82(1,35-2,46)$ & $1,52(1,15-2,01)$ \\
\hline Não & $98(25,9)$ & & 1,00 & 1,00 \\
\hline Doença pulmonar & & 0,121 & & --- \\
\hline Sim & $16(40,0)$ & & $1,47(0,96-2,24)$ & \\
\hline Não & $123(28,3)$ & & 1,00 & \\
\hline Depressão & & 0,001 & & \\
\hline $\mathrm{Sim}$ & $68(38,6)$ & & $1,53(1,14-2,05)$ & $1,42(1,08-1,87)$ \\
\hline Não & $71(24,1)$ & & 1,00 & 1,00 \\
\hline Demência & & 0,060 & & --- \\
\hline $\operatorname{Sim}$ & $78(33,1)$ & & $1,24(0,92-1,67)$ & \\
\hline Não & $60(25,2)$ & & 1,00 & \\
\hline Incontinência urinária & & 0,016 & & --- \\
\hline $\operatorname{Sim}$ & $102(33,0)$ & & $1,45(1,03-2,04)$ & \\
\hline Não & $37(22,4)$ & & 1,00 & \\
\hline
\end{tabular}


Continuação da Tabela 3

\begin{tabular}{|c|c|c|c|c|}
\hline Variável & $\mathrm{n}(\%)$ & $p^{*}$ & $\mathrm{RP} * *(\mathrm{IC} 95 \%)$ & $\mathrm{RP} * * *(\mathrm{IC} 95 \%)$ \\
\hline Incontinência fecal & & 0,063 & & --- \\
\hline $\operatorname{Sim}$ & $74(33,5)$ & & $1,22(0,91-1,65)$ & \\
\hline Não & $65(25,7)$ & & 1,00 & \\
\hline Multimorbidade & & 0,109 & & --- \\
\hline Sim & $90(31,8)$ & & $1,18(0,86-1,61)$ & \\
\hline Não & $48(25,0)$ & & 1,00 & \\
\hline Internação hospitalar & & 0,008 & & \\
\hline Sim & $56(37,8)$ & & $1,52(1,13-2,05)$ & $1,36(1,02-1,80)$ \\
\hline Não & $83(25,8)$ & & 1,00 & 1,00 \\
\hline MPI & & $<0,001$ & & \\
\hline $\operatorname{Sim}$ & $72(45,6)$ & & $2,43(1,81-3,27)$ & $2,13(1,60-2,83)$ \\
\hline Não & $54(18,7)$ & & 1,00 & 1,00 \\
\hline
\end{tabular}

*p: Teste qui-quadrado de Pearson; **RP: Razão de Prevalência bruta, Regressão de Poisson com variância robusta; ***RP: Razão de Prevalência ajustada, Regressão de Poisson com variância robusta.

No modelo final permaneceram associadas à polifarmácia excessiva ter cardiopatia $(\mathrm{RP}=1,40$; IC95\% 1,03-1,91), diabetes mellitus ( $\mathrm{RP}=1,52$; IC95\% 1,15-2,01), depressão (RP=1,42; IC95\% 1,08-1,87), história de internação hospitalar no último ano (RP=1,36; IC95\% 1,02-1,80) e fazer uso de MPI $(\mathrm{RP}=2,13$; IC95\% 1,60-2,83) (Tabela 3).

\section{DISCUSSÃO}

O presente estudo examina a prevalência e os fatores relacionados à polifarmácia excessiva entre pessoas idosas residentes em ILPI do Sul do Brasil. Os resultados sugerem que a polifarmácia excessiva é comum na população estudada, com uma em cada quatro pessoas idosas recebendo 10 ou mais fármacos, e que não apenas doenças crônicas como cardiopatia, diabetes mellitus e depressão, mas também a história de internação hospitalar no último ano e o uso de MPI estão a ela associados.

A prevalência do uso concomitante de 10 ou mais medicamentos, neste estudo, foi superior à verificada entre pessoas idosas residentes em casas de repouso da Europa, 24,3\% e da França, 21,1\% ${ }^{9}$. Outros estudos com pessoas idosas institucionalizadas, encontraram maiores prevalências de polifarmácia excessiva, como no exemplo sueco, $35,5 \%{ }^{1}$ e no suíço $44,1 \% 0^{11}$.
No contexto brasileiro, estudos investigaram o uso de polifarmácia (cinco ou mais medicamentos) entre pessoas idosas residentes em ILPI e verificaram prevalências de 27,5\%, em investigação que incluiu ILPI do Rio de Janeiro, Minas Gerais, Mato Grosso e Mato Grosso do $\mathrm{Sul}^{7}$ e $73,9 \%$ no estado de $\mathrm{SP}^{8}$. No entanto, não foi encontrada publicação que analisasse o uso simultâneo de 10 ou mais medicamentos por pessoas idosas institucionalizadas. Apenas um estudo com pessoas idosas da comunidade, atendidas em duas unidades básicas de saúde, em Minas Gerais, analisou o uso de polifarmácia excessiva, no qual 4,8\% das pessoas idosas faziam uso de 10 ou mais medicamentos ${ }^{14}$, percentual bastante inferior ao verificado neste estudo, o que poderia ser justificado pelo fato de pessoas idosas institucionalizadas geralmente serem mais frágeis, possuírem maior número de doenças crônicas e usarem mais medicamentos em comparação com as da comunidade ${ }^{1-4}$. Contudo, é prudente considerar que as distintas metodologias empregadas nos estudos, além de influenciar a prevalência de polifarmácia excessiva, tornam difíceis as comparações.

As diferenças na prevalência de polifarmácia excessiva entre idosos institucionalizados de diferentes contextos, podem ser influenciadas pelas distintas posturas dos prescritores, frente ao desafio de tratar pacientes complexos ${ }^{4}$. Enquanto alguns adotam condutas terapêuticas baseadas em diretrizes 
ou protocolos vigentes no país, outros podem, por exemplo, considerar as características e preferências da pessoa idosa ${ }^{2,4}$ como base para a recomendação terapêutica, o que poderia resultar em diferentes quantitativos de medicamentos prescritos.

Nessa perspectiva, um fator que poderia contribuir para a utilização de polifarmácia excessiva por pessoas idosas institucionalizadas do Sul do país, é o crescente uso de protocolos clínicos e diretrizes terapêuticas, como base para a conduta médica. No Brasil, o Ministério da Saúde recomenda às equipes de atenção básica, que se constituem na porta de entrada das pessoas idosas para o sistema de saúde, o uso de diretrizes clínicas baseadas em evidências para o tratamento da pessoa com doença crônica $^{19}$. Tradicionalmente essas diretrizes são baseadas em doenças únicas e não consideram a complexidade do indivíduo com multimorbidade, motivo pelo qual, pacientes idosos com doenças coexistentes, podem receber prescrições de vários medicamentos ${ }^{2}$. Essas evidências estão alinhadas aos nossos achados, visto que, a maioria das pessoas idosas usuárias de polifarmácia excessiva, possuíam também multimorbidade.

Ainda, questões relativas ao sistema de saúde de cada país, podem estar na gênese de tais diferenças. No sistema de saúde brasileiro, a implementação de políticas públicas, nos últimos anos, com vistas à garantia do tratamento e controle de doenças prevalentes no país, permitiu a ampliação do acesso aos medicamentos ${ }^{20}$, com destaque para a região Sul, na qual é registrado o maior percentual de acesso à assistência farmacêutica do país ${ }^{21}$. Nessa região também se verifica o maior percentual de pessoas idosas em uso de polifarmácia, 25,0\% contra somente $3,0 \%$ na região Norte $^{22}$. Essas evidências levam a crer, que a polifarmácia excessiva, verificada neste estudo, poderia ser favorecida pela maior facilidade de acesso aos medicamentos, pois, pessoas com cobertura gratuita de medicamentos, mostram um maior risco de polifarmácia em comparação com aqueles que precisam custear a assistência farmacêutica ${ }^{23,24}$. Corrobora ainda essa hipótese, o fato de que no presente estudo, os medicamentos usados com mais frequência por pessoas idosas em polifarmácia excessiva, quais sejam omeprazol e quetiapina, fazem parte da Relação Nacional de Medicamentos Essenciais e são, portanto, disponibilizados gratuitamente pelo Sistema Único de Saúde ${ }^{20}$.

Neste estudo, a polifarmácia excessiva mostrou-se associada à cardiopatia, diabetes mellitus e depressão. Outros estudos verificaram a mesma associação ${ }^{4,23}$. Tais doenças são de elevada prevalência entre pessoas idosas, independente do contexto onde vivem $^{4,23}$ e comumente tratadas com a combinação de fármacos ${ }^{23,25}$.

Contraintuitivamente, neste estudo, não se identificou associação entre polifarmácia excessiva e multimorbidade. Esse achado contrasta com a literatura acerca do tema ${ }^{5,6}$ e pode ser justificado pelo fato de que, na população estudada, as principais substâncias responsáveis pela polifarmácia excessiva foram o omeprazol e a quetiapina, e o uso desses medicamentos não indica, necessariamente, a presença de condição crônica ${ }^{26,27}$.

Entre os estudados, a polifarmácia excessiva se mostrou associada à internação hospitalar no último ano. Esse resultado é apoiado pela literatura ${ }^{3,22,28}$. Investigação conduzida entre pessoas idosas hospitalizadas no Paquistão, verificou que pacientes em uso de polifarmácia excessiva tiveram razão de chances 37 vezes maior de hospitalização em comparação com os não expostos à polifarmácia ${ }^{28}$. $\mathrm{O}$ uso de múltiplos medicamentos pode, de fato, acarretar danos capazes de determinar uma hospitalização ${ }^{5}$ e quanto maior o número de medicamentos em uso, maior a probabilidade de eventos adversos, que podem ser graves e fatais ${ }^{1}$.

No Brasil, as hospitalizações relacionadas a danos provocados por medicamentos, apresentam tendência crescente, sobretudo nas regiões Sul e Sudeste do país, com representatividade da população idosa. Pessoas idosas são mais susceptíveis a efeitos adversos, interações e toxicidade causada por medicamentos ${ }^{29}$ o que poderia contribuir para a necessidade de internação hospitalar nesse grupo e justificar a associação entre polifarmácia excessiva e hospitalização.

Outra possível explicação, poderia encontrar respaldo no fato de que a internação hospitalar, pressupõe o deslocamento da pessoa idosa através 
de diferentes configurações de cuidados e, esse movimento, poderia favorecer a ocorrência de inconsistências na lista de medicamentos em uso pela pessoa idosa. Dados da Organização Mundial de Saúde revelam que a maioria das pessoas idosas institucionalizadas já foi vítima de inconsistências na lista de medicamentos ${ }^{30}$. Nesse contexto, é possível que medicamentos em uso e problemas de saúde pregressos, não sejam considerados para a instituição de novo esquema terapêutico, tornando pouco provável a identificação de efeitos adversos à medicamentos e possíveis interações. Assim, reações adversas podem ser interpretadas como novos problemas de saúde e tratadas com novos fármacos, dando início à cascata de prescrição, fator que poderia contribuir para o uso de polifarmácia excessiva ${ }^{25}$.

Neste estudo a polifarmácia excessiva se mostrou associada ao uso de MPI. Nossos achados estão alinhados à literatura ${ }^{1,11,31}$. Entre os expostos à polifarmácia excessiva, a maioria fazia uso de MPI, enquanto no grupo não exposto, esse percentual reduziu-se pela metade. Evidências sugerem, de fato, que quanto maior o número de medicamentos prescritos, maiores são as chances de se receber um $\mathrm{MPI}^{11,28,31}$.

Esses resultados apontam para uma condição preocupante, pois indicam elevada prevalência de uso de MPI entre pessoas idosas institucionalizadas em uso de polifarmácia excessiva, o que deve ser motivo de alerta para profissionais e gestores, visto que, os riscos de efeitos nocivos desses medicamentos podem exceder os benefícios ${ }^{15}$. No entanto, apesar da relevante preocupação com os resultados negativos associados ao uso de MPI, a literatura demonstra que a prescrição desses medicamentos é frequente e aponta para um aumento da prevalência ao longo do tempo ${ }^{9,11}$, o que indica a necessidade de intervenções com vistas à redução da prescrição de MPI.

Como pontos fortes destacamos: o período considerado para a análise do uso de medicamentos, que envolveu os três meses anteriores à pesquisa, $\mathrm{O}$ que poderia reduzir a possibilidade de subestimação da polifarmácia excessiva, pois medicamentos podem ser usados semanalmente ou mensalmente; foram coletadas informações sobre o estado de saúde o que permite estudar fatores associados à polifarmácia excessiva; as informações relativas ao uso de medicamentos, ao estado de saúde e à presença de doenças crônicas foram obtidas por meio da análise dos prontuários, o que contribui para a fidedignidade dos achados.

Este estudo apresenta algumas limitações: o desenho transversal desta pesquisa não permite estabelecer uma relação de causa e efeito; o percentual de recusas pode ter ocorrido em ILPI com pessoas idosas com perfil de saúde diferente da amostra investigada, influenciando nos resultados do estudo, assim como a taxa de não resposta em algumas variáveis; alguns medicamentos classificados como um produto podem conter mais de uma substância química, e assim, o usuário ter sido erroneamente classificado como não polifarmácia excessiva, o que poderia contribuir para a subestimação do desfecho. Por tratar-se de amostragem não probabilística, a generalização dos resultados fica prejudicada, entretanto, os achados são similares aos encontrados na literatura científica.

\section{CONCLUSÃO}

Nossos achados seguem a tendência observada internacionalmente e sugerem que as doenças prevalentes entre pessoas idosas, a hospitalização e o uso de medicamentos potencialmente inapropriados são fatores para o uso de polifarmácia excessiva. Dos fatores associados, a maioria enquadra-se no grupo dos não modificáveis, como é o caso das doenças crônicas. No entanto, o uso de medicamentos potencialmente inapropriados para idosos é um fator passível de modificação, pois, com frequência, alternativas mais seguras e eficazes estão disponíveis. Desse modo, tornam-se necessárias ações com vistas a otimização da farmacoterapia prescrita às pessoas idosas institucionalizadas. Intervenções voltadas à capacitação e à educação continuada das equipes que atuam nas instituições, contemplando as especificidades da área da geriatria e da gerontologia, poderiam contribuir para a qualidade da terapia medicamentosa recomendada às pessoas idosas. Somado a isso, a revisão periódica das prescrições 
de medicamentos e a implementação de um sistema de referência e contrarreferência entre diferentes profissionais e serviços envolvidos na atenção à saúde da pessoa idosa são iniciativas com potencial para a redução da polifarmácia excessiva e do uso de medicamentos potencialmente inapropriados.

Editado por: Maria Luiza Diniz de Sousa Lopes

\section{REFERÊNCIAS}

1. Morin L, Johnell K, Laroche M-L, Fastbom J, Wastesson JW. The epidemiology of polypharmacy in older adults: register-based prospective cohort study. Clin Epidemiol. 2018;289-98. Disponível em: https://www.dovepress.com/the-epidemiology-ofpolypharmacy-in-older-adults-register-based-prospepeer-reviewed-article-CLEP.

2. Molokhia M, Majeed A. Current and future perspectives on the management of polypharmacy. BMC Fam Pract. 2017;18(1):1-10. Disponível em: http://bmcfampract.biomedcentral.com/ articles/10.1186/s12875-017-0642-0.

3. Walckiers D, Van der Heyden J, Tafforeau J. Factors associated with excessive polypharmacy in older people. Arch Public Health. 2015;73(1):1-10. Disponível em: http://www.archpublichealth.com/ content/73/1/50 .

4. Onder G, Liperoti R, Fialova D, Topinkova E, Tosato M, Danese P, et al. Polypharmacy in Nursing Home in Europe: results From the SHELTER Study. J Gerontol Ser A Biol Sci Med Sci. 2012;67(6):698-704. Disponível em: https://academic.oup.com/biomedgerontology/ article-lookup/doi/10.1093/gerona/glr233 .

5. World Health Organization. Medication Safety in Polypharmacy: Technical Report [Internet]. Geneva: WHO; 2019 [acesso em 10 out. 2020]. Disponível em:: https://www.who.int/publications/i/item/ medication-safety-in-polypharmacy-technical-report .

6. Khezrian M, McNeil CJ, Murray AD, Myint PK. An overview of prevalence, determinants and health outcomes of polypharmacy. Ther Adv Drug Saf. 2020;11:1-10. Disponível em: http://journals.sagepub. com/doi/10.1177/2042098620933741

7. Santiago LM, Luz LL, da Silva JFS, de Oliveira $\mathrm{PH}$, do Carmo CN, Mattos IE. Condições sociodemográficas e de saúde de idosos institucionalizados em cidades do Sudeste e Centro-Oeste do Brasil. Geriatr Gerontol Aging. 2016;10(2):86-92. Disponível em: http://www.ggaging.com/details/369/en-US/ socio-demographic-and-health-conditions-ofinstitutionalized-elders-in-cities-of-the-southeastand-middle-west-regions-of-brazil .
8. Garbin CAS, de Lima TJV, Araújo PC, Garbin AJI, Arcieri RM, Saliba O. Perfil da farmacoterapia utilizada por idosos institucionalizados. Arch Health Investig. 2017;6(7):322-7. Disponível em: http:// archhealthinvestigation.com.br/ArcHI/article/ view/2083 .

9. Herr M, Grondin H, Sanchez S, Armaingaud D, Blochet C, Vial A, et al. Polypharmacy and potentially inappropriate medications: a cross-sectional analysis among 451 nursing homes in France. Eur J Clin Pharmacol. 2017;73(5):601-8. Disponível em: http:// link.springer.com/10.1007/s00228-016-2193-z .

10. de Oliveira HSB, Corradi MLG. Aspectos farmacológicos do idoso: uma revisão integrativa de literatura. Rev Med. 2018;97(2):1-10. Disponível em: http://www.revistas.usp.br/revistadc/article/ view/140603.

11. Schneider R, Reinau D, Schur N, Blozik E, Früh $\mathrm{M}$, Signorell A, et al. Drug prescription patterns, polypharmacy and potentially inappropriate medication in Swiss nursing homes: a descriptive analysis based on claims data. Swiss Med Wkly. 2019;149:w20126. Disponível em: https://doi.emh.ch/ smw.2019.20126 .

12. DATASUS: Departamento de Informática do SUS [Internet]. Brasília, DF: DATASUS; 1991 - . Estudo de estimativas populacionais por município, idade e sexo 200-2015. 2017 [acesso em 13 out. 2020]. Disponível em: http://tabnet.datasus.gov.br/cgi/ deftohtm.exe?novapop/cnv/popbr.def.

13. World Health Organization. Collaboranting centre for Drug Statistics Methodology [Internet]. Oslo: WHOCC; 2020. ATC index with DDDs; [acesso em 13 out. 2020]. Disponível em: https://www.whocc.no/ atc_ddd_index_and_guidelines/atc_ddd_index/ .

14. de Oliveira PC, Silveira MR, Ceccato MDGB, Reis AMM, Pinto IVL, Reis EA. Prevalência e fatores associados à polifarmácia em idosos atendidos na Atenção Primária à Saúde em Belo Horizonte-MG, Brasil. Ciênc Saúde Colet. 2021;26(4):1553-64. Disponível em: http://www. scielo.br/scielo.php?script $=$ sci_arttext\&pid $=\mathrm{S} 1413$ 81232021000401553\&tlng=pt . 
15. Kuller L, Tonascia S. A follow-up study of the commission on chronic illness morbidity survey in Baltimore -IV. Factors influencing mortality from stroke and arteriosclerotic heart disease (1954-1967). J Chronic Dis. 1971;24(2-3):111-24.

16. American Geriatrics Society. American Geriatrics Society 2015 updated beers criteria for potentially inappropriate medication use in older adults. $\mathrm{J}$ Am Geriatr Soc. 2015;63(11):2227-46.

17. Bertolucci PHF, Brucki SMD, Campacci SR, Juliano Y. O Mini-Exame do Estado Mental em uma população geral: impacto da escolaridade. Arq Neuropsiquiatr. 1994;52(1):1-7. Disponível em: http://www.scielo.br/scielo.php?script $=$ sci_ arttext\&pid=S0004-282X1994000100001\&lng=pt\& tlng=pt

18. Lino VTS, Pereira SRM, Camacho LAB, Ribeiro Filho ST, Buksman S. Adaptação transcultural da Escala de Independência em Atividades da Vida Diária (Escala de Katz). Cad Saúde Pública. 2008;24(1):103-12. Disponível em: http://www.scielo. br/scielo.php?script $=$ sci_arttext\&pid=S0102-311X20 08000100010\&lng $=\mathrm{pt} \& \operatorname{lng}=\mathrm{pt}$.

19. Brasil. Ministério da Saúde. Secretaria de Atenção à Saúde, Departamento de Atenção Básica. Estratégias para o cuidado da pessoa com doença crônica [Internet]. Brasília, DF: MS; 2014 [acesso em 06 nov. 2020]. Disponível em: http://bvsms.saude.gov. br/bvs/publicacoes/estrategias_cuidado_pessoa_ doenca_cronica_cab35.pdf

20. Brasil. Ministério da Saúde, Secretaria de Ciência Tecnologia Inovação e Insumos em saúde, Departamento de Assistência Farmacêutica e Insumos Estratégicos. Relação Nacional de Medicamentos Essenciais - RENAME 2020 no âmbito do Sistema Único de Saúde (SUS) [Internet]. Brasília, DF: MS; 2020 [acesso em 20 nov. 2020]. Disponível em: http://bvsms.saude.gov.br/bvs/publicacoes/relacao_ medicamentos_rename_2020.pdf

21. Drummond ED, Simões TC, de Andrade FB. Acesso da população brasileira adulta a medicamentos prescritos. Rev Bras Epidemiol. 2018;21:e180007. Disponível em: http://www.scielo.br/scielo. php?script $=$ sci_arttext\&pid=S1415-790X2018000100 406\&lng $=\mathrm{pt} \& \mathrm{t} \operatorname{lng}=\mathrm{pt}$

22. Ramos LR, Tavares NUL, Bertoldi AD, Farias MR, Oliveira MA, Luiza VL, et al. Polypharmacy and Polymorbidity in Older Adults in Brazil: a public health challenge. Rev Saúde Pública. 2016;50(suppl 2):1-13. Disponível em: http://www. scielo.br/scielo.php?script $=$ sci_arttext\&pid $=$ S003489102016000300308\&lng=en\&tlng=en.
23. Marques PP, de Assumpção D, Rezende R, Neri AL, Francisco PMSB. Polypharmacy in communitybased older adults: results of the Fibra study. Rev Bras Geriatr Gerontol. 2019;22(5):1-10. Disponível em: http://www.scielo.br/scielo.php?script=sci_ arttext\&pid=S1809-98232019000500204\&tlng=en.

24. Richardson K, Kenny RA, Bennett K. The effect of free health care on polypharmacy: a comparison of propensity score methods and multivariable regression to account for confounding. Pharmacoepidemiol Drug Saf. 2014;23(6):656-65. Disponível em: http://www. ncbi.nlm.nih.gov/pubmed/24677639 .

25. Rochon PA, Gurwitz JH. The prescribing cascade revisited. Lancet. 2017;389(10081):1778-80. Disponível em: https://linkinghub.elsevier.com/retrieve/pii/ S0140673617311881 .

26. de Lima JM, Dal Fabbro AL, Funayama AR. Uso do omeprazol: estudo descritivo de pacientes idosos de uma Unidade de Saúde da Família (USF) de Ribeirão Preto, SP, Brasil. Infarma. 2019;31(1):46-53. Disponível em: http://revistas.cff.org.br/?journal $=$ infarma\&page $=$ article\&op $=$ view\&path[] $=2285$.

27. Müller L, Noseda R, Bertoli R, Bissig M, Ceschi A. Off-label use of quetiapine in nursing homes: Does medical specialty of prescribing physicians play a role? Br J Clin Pharmacol. 2020;86(7):1444-5. Disponível em: http://www.ncbi.nlm.nih.gov/pubmed/32100324 .

28. Sarwar MR, Dar AR, Mahar SY, Riaz T, Danish U, Iftikhar S. Assessment of prescribing potentially inappropriate medications listed in Beers criteria and its association with the unplanned hospitalization: a cross-sectional study in Lahore, Pakistan. Clin Interv Aging. 2018;13:1485-95. Disponível em: https://www.dovepress.com/assessment-ofprescribing-potentially-inappropriate-medicationslisted-peer-reviewed-article-CIA .

29. Santos GAS, Boing AC. Mortalidade e internações hospitalares por intoxicações e reações adversas a medicamentos no Brasil: análise de 2000 a 2014. Cad Saúde Pública. 2018;34(6):1-10. Disponível em: http:// www.scielo.br/scielo.php?script=sci_arttext\&pid=S0102311X2018000605011\&lng=pt\&tlng=pt .

30. World Health Organization. Medication Safety in Transitions of Care [Internet]. Geneva: WHO; 2019 [acesso em nov. 2020]. Disponível em: http://apps. who.int/bookorders.\%0Ahttps://www.who.int/ patientsafety/medication-safety/technical-reports/en/ .

31. Chang CB, Lai HY, Hwang SJ, Yang SY, Wu RS, Liu $\mathrm{HC}$, et al. Prescription of potentially inappropriate medication to older patients presenting to the emergency department: a nationally representative population study. Sci Rep. 2018;8(1):1-10. Disponível em: http://www. nature.com/articles/s41598-018-30184-4 . 